\title{
Glutamatergic dysfunction in catatonia? Successful treatment of three acute akinetic catatonic patients with the NMDA antagonist amantadine
}

\author{
G Northoff, J Eckert, J Fritze
}

\begin{abstract}
Therapeutic efficiacy of the NMDA antagonist amantadine is reported in three acute neuroleptic free akinetic catatonic patients. Intravenous infusion of amantadine led to the resolution of catatonic symptoms and considerable reductions of scores in various motor scales (Simpson Angus scale for extrapyramidal side effects (SEPS), the abnormal involuntary movement scale (AIMS), Rogers catatonia and schizophrenia scales). The therapeutic effect of amantadine showed a characteristic temporal pattern with most pronounced effects four to six hours after administration and recurrence of catatonic symptoms by 24 hours later, at least partially. Such a temporal pattern of therapeutic efficacy and decreasing efficacy occurred in all three patients on all days. The results suggest the central importance of glutamatergic dysfunction in catatonic syndrome.
\end{abstract}

(F Neurol Neurosurg Psychiatry 1997;62:404-406)

Keywords: catatonia; NMDA antagonist; glutamate; amantadine

Catatonia is nowadays regarded as a psychomotor syndrome exhibiting hypokinetic and hyperkinetic motor features as well as behavioural abnormalities. ${ }^{12}$ Due to the common association of the catatonic syndrome with schizophrenia and its symptomatic similarities with akinesia and rigidity in Parkinson's disease and neuroleptic malignant syndrome dopaminergic pathways may be of central importance. However, investigations of dopaminergic metabolism were inconsistent, showing either dopaminergic hyperfunction ${ }^{3}$ or normal function. ${ }^{4}$ Recently dysfunction of glutamatergic transmission has been suggested in schizophrenia, Parkinson's disease, and neuroleptic malignant syndrome..$^{5-7}$ There have been reports about therapeutic efficiacy of the NMDA antagonist amantadine in Parkinson's disease and neuroleptic malignant syndrome. ${ }^{5}$ Because of the similarities between catatonic syndrome, Parkinson's disease, and neuroleptic malignant syndrome with regard to akinesia and rigidity we treated three acute akinetic catatonic patients with intravenous amantadine.

\section{Methods}

All incoming patients in a period from 1 January 1993-31 December 1995 were screened for catatonic syndrome according to the criteria of Rosebush et al. ${ }^{8}$ In this period we found a $2 \cdot 7 \%$ incidence of patients with four or more catatonic symptoms and an $11 \cdot 2 \%$ incidence of patients with at least one catatonic symptom. For inclusion in our study patients had to fulfill at least four of 12 criteria and they had to be akinetic as evaluated by two independent raters (GN, JF). Catatonic patients with a history of paranoid schizophrenia were excluded because amantadine might have further exacerbated psychotic symptoms and in all three patients finally treated with amantadine this associated diagnosis was not clear on admission. Catatonic patients taking neuroleptic or other psychotropic drugs (for example, lorazepam or antidepressants) on admission were also excluded to avoid interference with amantadine $(n=6)$. Three acute akinetic catatonic patients fulfilling the appropriate conditions were selected for treatment. Each patient was initially given $500 \mathrm{mg}$ amantadine intravenously. If the patient was still catatonic or had become catatonic again on the next day the same dose was given 24 hours after the previous infusion. Treatment with amantadine was discontinued when the patient was no longer catatonic for at least eight to 10 hours. During treatment with amantadine no other psychotropic medication, such as neuroleptic drugs or lorazepam, was given. Effects of amantadine on psychomotor symptoms were evaluated with the Simpson Angus scale for extrapyramidal side effects (SEPS), ${ }^{9}$ the abnormal involuntary movement scale (AIMS), ${ }^{10}$ Rogers catatonia and schizophrenia scales, ${ }^{11}$ and the global assessment scale (GAS) ${ }^{12}$ before and six hours after treatment with amantadine on each day by an independent rater (JE). In addition, the catatonic syndrome criteria of Rosebush et $a^{8}$ were rated by another rater $(\mathrm{GN})$, before and 2,4 , $6,8,10$, and 12 hours after amantadine. On discharge diagnosis of the associated disease according to DSM IV ${ }^{13}$ was made by an independent doctor. 


\section{Results}

In all three patients, who were similar with regard to catatonic symptoms, amantadine led to considerable reductions in scores in SEPS, AIMS, and Rogers catatonia and schiophrenia scales six hours after administration (fig 1). Twenty four hours later catatonic symptoms recurred, at least partially, as can be seen on increasing scores on the following day before the next infusion (fig 1). Such a temporal pattern of therapeutic efficacy after six hours and decreasing efficacy after 24 hours could be seen in all three patients on all days.

In the next section detailed descriptions for each case are given.

PATIENT 1

A 25 year old neuroleptic naive student showed mutism, rigidity, akinesia, staring, catalepsy, posturing, negativisms, and autism on admission to hospital for the first time. Three hours after intravenous infusion of amantadine he was talking and moving fluently, showing no catatonic signs. Six to eight hours later his state gradually deteriorated such that he became catatonic again. The next day he predominantly showed hyperkinetic movements with mannerisms, stereotypies, and grimacing. Two to three hours after the second infusion of amantadine these symptoms were completely resolved and the patient showed no catatonic signs at all. Six to eight hours later he again became catatonic exhibiting posturing, akinesia, staring, and grimacing which lasted until the next day. He received a third infusion of amantadine which led to resolution of catatonic symptoms after three to four hours with no reccurrence. The patient reported delusions and auditory hallucinations such that on discharge he was diagnosed as having catatonic schizophrenia.

Figure 1 Scores before (A) and after (B) intravenous amantadine.

\section{PATIENT 2}

A 36 year old neuroleptic free mechanic showed mutism, akinesia, posturing, staring, rigidity, stereotypies, and autism on admission. Three hours after receiving intravenous amantadine all catatonic symptoms except mutism disappeared and he moved fluently. Six to eight hours later similiar catatonic symptoms recurred lasting until the next day. He was given a second infusion of amantadine which reversed all his catatonic symptoms including mutism, which did not reappear later. While in hospital he showed predominant negative symptoms and he was diagnosed as having schizophrenia. He said that he had stopped his neuroleptic drugs seven months before admission.

\section{PATIENT 3}

A 25 year old neuroleptic free worker was admitted to hospital for the first time, showing mutism, rigidity, akinesia, autism, grimacing, catalepsy, posturing, and negativisms. Four to five hours after infusion of amantadine catatonic symptoms disappeared almost completely except for a slight posturing which deteriorated some hours later. The next day he was catatonic again (akinesia, mutism, posturing, rigidity, autism, grimacing) and a second infusion of amantadine was given. This again led to resolution of catatonic symptoms after four to five hours with no recurrence. However, the patient became aggressive and anxious and lorazepam and haloperidol were given. While in hospital he showed delusions and auditory hallucinations and he was diagnosed as having catatonic schizophrenia.

\section{Discussion}

We report on the transient therapeutic efficacy of an NMDA antagonist in three patients with a homogeneous picture of acute akinetic catatonia. Our three patients showed the following similarities: (1) they did not exhibit any vegetative alterations or hyperthermia; (2) all three patients showed akinesia, behavioural abnormalities (negativisms, staring, autism, mutism), and hyperkinesiae (stereotypies, mannerisms); (3) amantadine led, in all three patients, to reversal of hypokinetic features and improvement in hyperkinesiae and behavioural abnormalities; (4) the therapeutic effect of amantadine followed a similar course in all three patients with most pronounced effects after four to six hours and gradually decreasing efficacy after 10-12 hours with partial reappearance of catatonic symptoms; (5) therapeutic effects of amantadine were shown to be reproducible by means of another infusion on the second day; (6) all three patients spontaneously reported (except the third patient on the second day) reductions of anxiety and tension after infusion of amantadine; (7) the patients were not taking neuroleptic or any other psychotropic drugs and the resolution of the catatonic symptoms can only be attributed to the effect of amantadine or to spontaneous remission, the second being unlikely given the timing of improvement; (8) all three catatonic 
patients were diagnosed as schizophrenic on discharge. Our findings suggest that the NMDA antagonist amantadine, which also releases dopamine, is therapeutically effective in the catatonic syndrome suggesting a glutamatergic or dopaminergic dysfunction; (9) no subjective or objective pharmacological side effects were seen. To our knowledge there have been no reports about glutamatergic neurotransmission or therapeutic efficiacy of NMDA antagonists in the catatonic syndrome.

Considering the symptomatic similarities between catatonia and Parkinson's disease and neuroleptic malignant symptom with regard to akinesia and rigidity it may be suggested that similiar glutamatergic hyperfunction occurs in the projections from the nucleus subthalamicus to the globus pallidus internus and for striatal dopaminergic hypofunction in catatonia. Such an assumption would explain the successful reversal of akinesia and rigidity in our patients but it could not account for the therapeutic effect on hyperkinesiae and behavioural abnormalities. However, the association of catatonia with schizophrenia is paradoxical as glutamatergic hypofunction has been postulated in schizophrenia. ${ }^{7}$ Thus administration of the NMDA antagonist and dopamine releaser amantadine should have worsened catatonic symptoms as suggested by the known therapeutic effect of partial indirect NMDA agonists in patients with chronic schizophrenia. ${ }^{14}$

We suggest the following hypothesis about glutamatergic and dopaminergic dysfunction

SMA = Supplementary motor area
DA = Dopamine
Glut = Glutamate
Asp = Aspartate

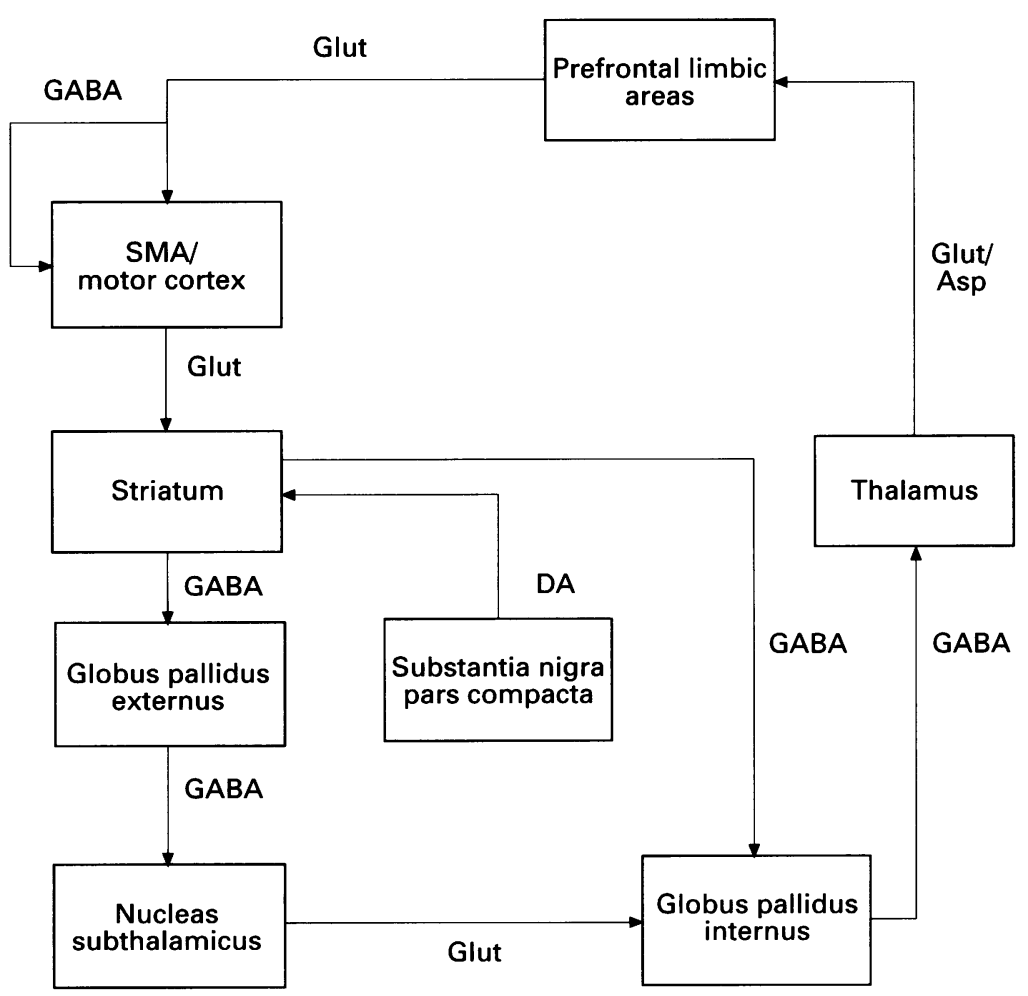

Figure 2 Glutamergic, GABAergic, and dopaminergic pathways. in catatonia: as in schizophrenia ${ }^{7}$ prefrontal and limbic glutamatergic pathways to the supplementary motor area (SMA) and motor cortex may be hypofunctional and may account for behavioural abnormalities in catatonia. ${ }^{2}$ The consecutive decreased release of $\gamma$-aminobutyric acid (GABA) in frontal interneurons, which in turn leads to less inhibition of glutamate, results in frontostriatal glutamatergic hyperfunction (fig 2). Such a hypothesis would be in accordance with the well known therapeutic efficacy of the GABA potentiator lorazepam in catatonia ${ }^{8}$ which may lead to GABA down regulation of frontostriatal glutamate.

Frontostriatal glutamatergic hyperfunction may lead via GABAergic pathways to increased glutamatergic function in the pathways from the nucleus subthalamicus to the globus pallidus internus resulting (similar to Parkinson's disease) in akinesia (fig 2). In our three catatonic patients the NMDA antagonist amantadine might have down regulated glutamatergic frontostriatal pathways either via NMDA antagonism or by releasing striatal dopamine, which in turn inhibits glutamate, in keeping with decreased presynaptic dopaminergic striatal function as seen in one catatonic patient. ${ }^{15}$ Due to the complexity of these networks such a hypothesis must remain speculative. GABAergic dysfunction in anterior cingulate areas $^{16}$ and alterations in other glutamate receptors (Ampa, Kainate, NMDA) may also be relevant in the catatonic syndrome. ${ }^{17}$

1 Gelenberg AJ. The catatonic syndrome. Lancet 1976;i:1339-41.

2 Taylor MA. Catatonia: a review of the behavioral neurologic syndrome. Neuropsychiatry, Neuropsychology and Behavioral Neurology 1990;3:48-72.

3 Northoff G, Demisch L, Wenke J, Pflug B. Plasma homovanillic acid concentration in catatonia. Biol Psychiatry 1996;39:436-43.

4 Starkstein S, Petracca G, Teson A, Chemerinski E, Merello $M$, Migliorelli R, Leiguardia R. Catatonia in depression: prevalence, clinical correlates, and validation of a scale. $\mathcal{F}$ Neurol Neurosurg Psychiatry 1996;60:326-32.

5 Kornhuber J, Weller M, Riederer P. Glutamate receptor antagonists for neuroleptic malignant syndrome and akinetic hyperthermic Parkinsonian crisis. F Neural Transm Park Dis Dement Sect 1993;6:63-72.

6 Greenamyre T Glutamate-dopamine interactions in the basal ganglia: relationship to Parkinson's disease. $f$ Neural Transm Gen Sect 1993;91:255-69.

7 Tsai G, Passani L, Slusher B, Carter R, Baer L, Kleinman J, Coyle J. Abnormal excitatory neurotransmitter metabolism in schizophrenic brains. Arch Gen Psychiatry 1995; lism in schizo.

8 Rosebush P, Furlong B, Mazurek M. Catatonic syndrome in a general psychiatric population: frequency, clinical presentation and response to lorazepam. $\mathcal{F}$ Clin Psychiatry 1990;9:357-62.

9 Simpson GM, Angus JWS. A rating scale for extrapyramidal side-effects. Acta Psychiatr Scand 1970;212(suppl):11-9.

10 Guy W, ed. ECDEU assessment manual for psychopharmacology. Washington, DC: ECDEU 1976;534-7.

11 Lund CE, Mortimer AM, Rogers D. Motor, volitional and behavioral disorders in schizophrenia. Br $\mathcal{F}$ Psychiatry 1991;158:323-7.

12 Endicott J, Spitzer R, Flies J, et al. The global assessment scale. Arch Gen Psychiatry 1976;33:766-71.

13 American Psychiatric Association. Diagnostic manual. 4th ed. Washington DC: APA, 1994

14 Goff DC, Tsai G, Manoach D, Coyle J. Dose-finding of Dcycloserine added to neuroleptics for negative symptoms cycloserine added to neuroleptics for negative symp.

15 Hietala I, Syvälahti E, Vuorio K, Räkköläinen V, Bergman J, Haaparanta $M$, et al. Presynaptic dopamine function in Haaparanta $M$, et al. Presynaptic dopamine function in
striatum of neuroleptic-naive schizophrenic patients.

16 Benes F, Vincent S, Alsterberg G, Bird E, Giovanni J. Increased GABA-A receptor binding in superficial layers of cingulate cortex in schizophrenia. $\mathcal{F}$ Neurosci 1992; 12:924-9.

17 Eastwood SL, Porter R, Burnet P, Kerwin R, Harrison P. Non-NMDA-glutamate receptor expression in schizophrenia. Schizophr Res 1996;18:174. 